\title{
Dream Content Changes in Women After Mastectomy: An Initial Study of Body Imagery After Body-Disfiguring Surgery
}

\author{
Alessandra Giordano, Valentina Francese, Elena Peila, Antonella Tribolo \\ Mario Airoldi, Riccardo Torta, Roberto Mutani, and Alessandro Cicolin \\ University of Torino and University Hospital "San Giovanni Battista-Molinette", \\ Torino, Italy
}

\begin{abstract}
The changes in body perception after invasive surgery and their internalization in dreams have been mostly neglected in the literature on dreams. We evaluated consecutive newly diagnosed patients with breast cancer about their dream content, sleep quality and quantity, and their mood both before and after the surgical intervention (TO and T1), to determine whether the loss of a body part with specific sexual features (such as the breast) could change body schema and actuate a sexual sphere restructuring. Dream content was coded according to the Hall and Van de Castle (1966) method. Results were analyzed to compare T0, T1, and normative data (DreamSat). In our sample, we found significant differences in the dreams between TO and T1 in some anatomical elements (torso anatomy self-concept). We also found differences between patients (T0 and T1) and the normative data, with an increased presence of male and family characters, the proportion of dead people, striving elements with failure, and a decreased presence of friends, aggressiveness, and familiar settings in the patients' dreams. Our results demonstrate that dream content in patients who have undergone cancer treatment for breast cancer is different from that of normal subjects and that there is an adjustment of dream content in patients after surgery. These results support Domhoff's hypothesis about continuity between dreams and waking life, in the sense that "the concerns people express in their dreams are the concerns they have in waking life (Domhoff, 2003).
\end{abstract}

Keywords: dreaming, dream content, mastectomy, breast cancer, sleep

Alessandra Giordano, Valentina Francese, Elena Peila, Antonella Tribolo, Roberto Mutani, and Alessandro Cicolin, Department of Neuroscience, University of Torino, and University Hospital "San GiovanniBattista-Molinette", Torino, Italy; Mario Airoldi, Department of Oncology, University of Torino and University Hospital "San Giovanni Battista-Molinette," Torino, Italy; Riccardo Torta, Clinical and Oncologic Psychology Unit, Department of Neuroscience, University of Torino and University Hospital "San GiovanniBattista-Molinette", Torino, Italy.

We declare that we have no personal or financial support or involvement with organization(s) with financial interest in the subject matter of the paper, or any actual or potential conflict of interest.

Correspondence concerning this article should be addressed to Alessandro Cicolin, Sleep Disorder Center, Department of Neurosciences, University of Torino, Via Cherasco 15, 10126 Torino, Italy. E-mail: alessandro.cicolin@unito.it 
As long as Freud's interpretive theory prevailed in the study of dreams, the relation between dreaming and waking conceptions was considered to be mediated by the censure of latent content, with the meaning accessible only through psychoanalytical interpretation. More recent neurobiological perspectives demonstrate a consistently different point of view. According to Hobson's (1992) dream conceptualization, for example, the meaning of dreams should be considered clearly "transparent" and not elaborated. He said that there are common formal aspects concerning everybody's dreams, and there are some content differences related to age, gender, personality characteristics, and cross-cultural differences.

Consistent with Hobson's view, Calvin S. Hall and Robert L. Van de Castle (1966) developed a specific coding system to study dream content. They assumed that "the frequency with which a dream element appears reveals the concerns and interests of the dreamer," providing the opportunity "to link dream content with the waking thoughts and behavior of the dreamer" (Domhoff, 1996). Domhoff, applying the Hall and Van de Castle (1966) coding system with healthy subjects, demonstrated that dream recall is largely independent from personality and cognitive variables and that age and gender variables are stable during adulthood (Domhoff, 1996; 2001). He hypothesized a continuity between dreams and waking life, in the sense that "the concerns people express in their dreams are the concerns they have in waking life" (Domhoff, 2003). The Hall and Van de Castle (1966) method has also been clinically applied (Newton, 1970), in particular, to a study of dream content in sleep disorders (Fantini et al., 2005).

Following Domhoff's hypothesis, the analysis of dream reports would be useful for evaluating the changes of mental body image and self-perception in dreams after patients have experienced different kinds of disease-implicating body disfigurements. Even though many studies have evaluated dream content in healthy volunteers (for reviews on the topic, see Domhoff, 1996, 2003, 2007; Domhoff, Meyer-Gomes, \& Schredl, 2006), few authors have evaluated the dreams of paralyzed or amputated patients (Mulder, Hochstenbach, Dijkstra, \& Geertzen, 2008; Newton, 1970; Brugger, 2008; Vetrugno, Arnulf, \& Montagna, 2008). Disfigurements related to cancer treatments force patients to deal with psychological distress, the loss of their previous appearance, and the alteration of their body image (Rumsey \& Harcourt, 2004). In particular, previous authors have stressed that breast cancer diagnosis and treatment significantly affect body image and psychosocial adjustment (Carver et al., 1998; Sheppard \& Ely, 2008; Schover et al., 1995), relationships with partner and children (Anllo, 2000; Walsh, Manuel, \& Avis, 2005) and sexuality (Schover et al., 1995; Henson, 2002; Anllo, 2000; Ghizzani, Pirtoli, Bellezza, \& Velicogna, 1995; Sheppard \& Ely, 2008). Nevertheless, these authors have not evaluated changes in dream content.

The aim of this study is to assess dream content in patients with breast cancer who are undergoing a mastectomy, a surgery potentially producing profound body modifications.

\section{MATERIALS AND METHODS}

For three months, all consecutive outpatients referred to the Department of Oncology in the University Hospital "San Giovanni Battista" of Torino (Italy) were 
evaluated. Inclusion criteria were the following: age between 30 and 70 years, primary breast cancer diagnosis in the previous 3 months, in physiological or pharmacologically induced menopause, and awaiting breast resection surgery. The exclusion criteria were impaired cognitive ability (MMSE $<24$ ), taking of drugs that could affect REM sleep (i.e., antidepressants and benzodiazepines), and the presence of psychiatric and sleep disorders according to the Diagnostic and Statistical Manual of Mental Disorders, Fourth Edition, Text Revision (DSM-IV-TR; American Psychiatric Association, 2000) and the International Classification of Sleep Disorders, Second Edition (ICSD-2; American Academy of Sleep Medicine, 2005). The Local Ethical Committee approved the protocol, and all the patients signed the informed consent form.

Patients were evaluated at T0 (7-10 days before the surgery) and at T1 (3 months after mastectomy). The T0 evaluation included the following: (a) demographic (age, instruction level, employment, family) and clinical (hand preference, drug use, quantity and quality of sleep, oncologic diagnosis) data; (b) a dream report form (DRF; Domhoff, 1996) in which the patients were asked to describe five recent dreams; (c) the Dream Remind Questionnaire (DRQ), a multiplechoice, self-description test administered to obtain people's explicit knowledge of their dreams, in which the dreamer judges the more usual dreaming ambience, scene, self, and emotions on a Likert scale (Rezzonico \& Liccione, 2004); (d) the Beck Depression Inventory (BDI; Beck, 1959); and (e) the State Trait Anxiety Inventory, Form Y (STAI; Spielberg, 1983), which involves self-administered scales to assess mood and anxiety, respectively. The T1 evaluation included the following: the DRF, DRQ, BDI, and STAI.

DRFs were coded according to the Hall and Van de Castle (1966) coding system. Dreams were blindly scored (mixing T0 and T1 dream reports) by a sleep medicine board-certified psychologist; difficult or ambiguous issues were resolved in group discussions. The alphanumeric codes were uploaded into a DreamSAT spreadsheet, (free web version), which provided frequencies for the total series of dream reports and percentage calculations for T0 compared to T1 (Domhoff \& Schneider, 1998; Domhoff, 1996). Coded dream data also was statistically compared to normative data using Adam Schneider's DreamSAT (available at www.dreamresearch.net).

Other variables were analyzed by SPSS 17.0 for Windows, using a $t$ test and the chi-square test $\left(\chi^{2}\right)$. Results are presented in terms of mean $\pm S D$ with significance levels at $p<.05$.

\section{RESULTS}

Out of 66 eligible outpatients, 20 did not fulfill inclusion criteria, 16 refused to take part in the study (inability to recall dreams and excessive emotional tension), and 15 dropped out (withdrew consent between T0 and T1). Fifteen subjects completed the study (mean age $=51 \pm 11$ years, education $=10 \pm 4$ years)

After mastectomy, the total sleep time was significantly increased, $\mathrm{T} 0=5.8 \pm 1.7 \mathrm{hr}, \mathrm{T} 1=6.5 \pm 0.9 \mathrm{hr}, p \leq .05$, and the sleep quality was unchanged (sufficient/good in 10/14 patients both at T0 and T1). STAI and BDI scores were similar at T0 and T1 (STAI Y1 T0 = $24.4 \pm 16.4, \mathrm{~T} 1=21.0 \pm 11.7, n s$; STAI Y2 
$\mathrm{T} 0=19.5 \pm 11.4, \mathrm{~T} 1=18.2 \pm 9.7, n s ; \mathrm{BDI} \mathrm{T} 0=9.1 \pm 7.4 ; \mathrm{T} 1=8.2 \pm 6.5, n s)$, with a slight (nonsignificant) reduction at $\mathrm{T} 1$.

Evaluating the dreamers' explicit knowledge (DRQ; see Table 1), we found at $\mathrm{T} 1$ a statistically significant increase in stated presence of wise self, determined self, curiosity, anger, and shame. On the other hand, excitement was reduced in T1. We also found some changes not statistically significant: At T1, there is a reduction of erotic and violent environments, frightened-self perceptions, and repulsion, along with a slight increase of loved and powerful self-perception.

Evaluating the DRF, 83 dreams were collected (47 at T0 and 36 at T1) and analyzed using the Hall and Van de Castle (1966) coding method. When compared with normative data (see Table 2), oncologic patients showed increased presence of male and family characters, a higher proportion of dead people, striving elements with failure, and decreased presence of friends, aggressiveness, and familiar settings in their dreams at both T0 and T1. Moreover, oncologic patients showed more misfortunes at $\mathrm{T} 1$.

Comparing dream contents at $\mathrm{T} 0$ versus $\mathrm{T} 1$, we found a significant increase of torso-anatomy percent, one of five self-concept percents in the Hall and Van de Castle (1966) system (see Table 2).

We also discovered that dream contents at $\mathrm{T} 1$ are more similar to norms than they are at T0. The number of the most recent, recurrent and negative dreams was the same both before and after the surgery, while the number of positive dreams was higher before the surgery, and sexual dreams were poorly reported in both periods, especially after the surgery.

\section{DISCUSSION}

Our study analyzed dream content with the Hall and Van de Castle (1966) method in patients who underwent surgery for breast cancer in order to understand the possible internalization of body modification. Our findings are consistent with Domhoff's hypothesis about continuity between dreams and waking. To our knowledge, no other investigation on body modification perception in dreams using the Hall and Van de Castle (1966) coding system has been completed in oncologic patients. Moreover, few studies have analyzed dream content in people who have undergone surgery that impacts body image. Mulder et al. (2008), using no standardized dream coding method, reported that amputees continue to dream about their body as intact 12 years after the amputation.

Table 1. Paired Sample t Test Between T0 and T1 for Significant DRQ items

\begin{tabular}{lccccc}
\hline \multicolumn{1}{c}{ DRQ } & T0 $(M)$ & T0 $(S D)$ & T1 $(M)$ & T1 $(S D)$ & $p$ \\
\hline Wise self & 2.8 & 1.6 & 4.2 & 1.6 & $\leq 0.05$ \\
Determined self & 2.7 & 1.6 & 4.0 & 1.6 & $\leq 0.05$ \\
Excitement & 2.8 & 1.5 & 2.1 & 1.4 & $\leq 0.05$ \\
Curiosity & 2.8 & 1.5 & 4.3 & 1.3 & $\leq 0.05$ \\
Anger & 2.3 & 1.9 & 2.8 & 1.7 & $\leq 0.05$ \\
Shame & 1.2 & 0.6 & 1.7 & 1.0 & $\leq 0.05$
\end{tabular}

Note. $\mathrm{T} 0=7-10$ days before surgery; $\mathrm{T} 1=3$ months after mastectomy; DRQ $=$ Dream Remind Questionnaire. 
Table 2. Dream Characteristics of Oncologic Patients (at T0 and T1) and Norms

\begin{tabular}{|c|c|c|c|c|c|c|}
\hline DreamSAT categories & T0 & $\mathrm{T} 1$ & $\begin{array}{c}\text { Norms } \\
\text { (female) }\end{array}$ & $\begin{array}{c}\text { T0 vs. } \\
\text { norms }(p)\end{array}$ & $\begin{array}{c}\text { T1 vs. } \\
\text { norms }(p)\end{array}$ & $\begin{array}{l}\text { T0 vs. } \\
\text { T1 }(p)\end{array}$ \\
\hline \multicolumn{7}{|l|}{ Characters } \\
\hline Male/female \% & 65 & 79 & 48 & $\leq 0.05$ & $\leq 0.05$ & $n s$ \\
\hline Familiarity \% & 77 & 62 & 58 & $\leq 0.05$ & $n s$ & $n s$ \\
\hline Friends \% & 13 & 5 & 37 & $\leq 0.05$ & $\leq 0.05$ & $n s$ \\
\hline Relative \% & 58 & 56 & 19 & $\leq 0.05$ & $\leq 0.05$ & $n s$ \\
\hline Dead and imaginary \% & 20 & 15 & 1 & $\leq 0.05$ & $\leq 0.05$ & $n s$ \\
\hline Animal \% & 6 & 7 & 4 & $n s$ & $n s$ & $n s$ \\
\hline \multicolumn{7}{|l|}{ Social interaction } \\
\hline Aggression/friendliness \% & 32 & 45 & 51 & $\leq 0.05$ & $n s$ & $n s$ \\
\hline Befriender \% & 31 & 55 & 47 & $n s$ & $n s$ & $n s$ \\
\hline Aggressor \% & 25 & 11 & 33 & $n s$ & $n s$ & $n s$ \\
\hline Physical aggression \% & 22 & 27 & 34 & $n s$ & $n s$ & $n s$ \\
\hline \multicolumn{7}{|l|}{ Settings } \\
\hline Indoor setting \% & 66 & 57 & 61 & $n s$ & $n s$ & $n s$ \\
\hline Familiar setting \% & 56 & 30 & 79 & $\leq 0.05$ & $\leq 0.05$ & $n s$ \\
\hline \multicolumn{7}{|l|}{ Self-concept } \\
\hline Self-negativity \% & 65 & 75 & 66 & $n s$ & $n s$ & $n s$ \\
\hline Bodily misfortunes \% & 27 & 33 & 35 & $n s$ & $n s$ & $n s$ \\
\hline Negative emotions \% & 62 & 79 & 80 & $n s$ & $n s$ & $n s$ \\
\hline Dreamer-involved success \% & 33 & 32 & 42 & $n s$ & $n s$ & $n s$ \\
\hline Torso/anatomy \% & 6 & 33 & 20 & $n s$ & $n s$ & $\leq 0.05$ \\
\hline \multicolumn{7}{|l|}{ Dreams with at least one } \\
\hline Aggression \% & 15 & 16 & 44 & $\leq 0.05$ & $\leq 0.05$ & $n s$ \\
\hline Friendliness \% & 36 & 32 & 42 & $n s$ & $n s$ & $n s$ \\
\hline Sexuality \% & 13 & 5 & 4 & $n s$ & $n s$ & $n s$ \\
\hline Misfortune \% & 40 & 51 & 33 & $n s$ & $\leq 0.05$ & $n s$ \\
\hline Good fortune \% & 13 & 8 & 6 & $n s$ & $n s$ & $n s$ \\
\hline Success \% & 19 & 16 & 8 & $n s$ & $n s$ & $n s$ \\
\hline Failure \% & 30 & 30 & 10 & $\leq 0.05$ & $\leq 0.05$ & $n s$ \\
\hline Striving \% & 43 & 41 & 15 & $\leq 0.05$ & $\leq 0.05$ & $n s$ \\
\hline
\end{tabular}

Note. $\mathrm{T} 0=7-10$ days before surgery; $\mathrm{T} 1=3$ months after mastectomy.

A study focused on the amount of physical activity in dreams of paralyzed people highlights that, compared to norms, the kinesthetic dream content is increased in recently paralyzed men and reduced in long-term paralyzed men (Newton, 1970).

We also analyzed changes in sleep quality and quantity, emotional aspects (STAI and BDI), and explicit dream knowledge (DRQ). Our results demonstrate that sleep, explicit dream knowledge, and dream content changed during the course of the therapeutic time period considered in this article. Moreover, we found a total sleep time (TST) improvement without significant changes of sleep quality. The increase of TST from a partial deprivation (less than $6 \mathrm{hr}$ per night) at T0 to a nearly normal condition ( $6 \mathrm{hr}$ and a half per night) at T1 could be related to anxiety about the imminent surgery, while after mastectomy our patients were less anxious and depressed, as revealed by the slight reduction of STAI and BDI scores at T1, even if some patients (4/14) were still in a troubled posttraumatic condition with poor quality of sleep.

The differences in explicit dream knowledge between T0 and T1 seem to develop in two distinct areas. At first, emotions, self, and setting concepts seem to reveal patients' worries: the lowering of excited emotions and erotic settings, 
from $\mathrm{T} 0$ to $\mathrm{T} 1$, in combination with the increase of anger and shame, could reflect perception of oneself as less attractive, suggesting the mastectomy's influence on the subjects' sexual sphere. Nevertheless, the dreamers' self, in fact, appears less frightened and disturbed by repulsion and more aware, determined, loved, and curious after the surgery. Such changes are probably also related to mood and anxiety improvement. This self-concept modification suggests a deeper self-restructuring appearing at $\mathrm{T} 1$, which could be tied to the ordeal of overcoming cancer and removing pain and evil.

These results agree with literature concerning women's waking thoughts after mastectomy. As Sheppard and Ely (2008) summarized in their literature review, breast cancer diagnosis and mastectomy greatly impact patients' sexuality and produce changes in the relationships with their partner and children (Walsh et al., 2005). Moreover, disfigurements in general have profound impact upon individual body-image concern, self-confidence, social interactions (Rumsey \& Harcourt, 2004), and self-consciousness about one's appearance (Shover et al., 1995; Walsh et al., 2005). For example, there are fears of abandonment that characterize breast cancer patients' waking thoughts [Anllo, 2000], which may reflect the dreams of abandonment frequently reported by the youngest women in our sample. This is not surprising, given that the reaction to the diagnosis and treatment of breast cancer depends on a women's age, as acceptance of breast illness and scars are less difficult for older patients (Ghizzani et al., 1995). The self-perception of being less attractive that we found in dreams is related to specific problems in daily life: $45 \%$ of all breast cancer women feel anxious, depressed and less attractive, experiencing a loss of desire related to an "altered body image due to loss or alteration in breast tissue, hair loss, pain, altered child-bearing capacity, and change in health status perception" (Henson, 2002, 262).

Dream content modifications between $\mathrm{T} 0$ and $\mathrm{T} 1$ are specifically related to body image. Our patients reported more body-related topics in dreaming after mastectomy. Before surgery, torso-anatomy self-concepts were clearly neglected (healthy people seemed to have no need to explore that specific part of their body as demonstrated by normative data). It, therefore, could be hypothesized that the imminence of the surgery seems to drive our patients not to think about their bodies, in particular about their breasts, maybe to protect themselves from fear (Anllo, 2000). DreamSAT analysis highlighted differences in oncologic patients when compared to normative data, both at T0 and T1. In fact, oncologic patients showed an increased proportion of male and relative characters, dead people, striving and failure elements, and decreased presence of friends, aggressiveness and familiar settings in their dreams. According to Domhoff's perspective, a lack of friends, as opposed to an abundance of family members, and increased misfortunes could be related to a dreamer's maladjustment problems. With regard to the high proportion of strivings elements that result in failure, they could be tied to a simulation of threatening events, following Revonsuo's (2000) evolutionary hypothesis, which maintains that the function of dreaming is to rehearse threat perception and threat avoidance. The fact that the proportion of dead characters is significantly greater than norms both before and after the surgery probably reflects the patients' worries about the clinical outcome, and the low aggressiveness might reflect a general sense of resignation. 
The high presence of misfortunes found in the study sample at T1 probably reflects and enhances dreamers' daily worries, as explained by Revonsuo's (2000) perspective and Ernest Hartmann's (1996) theory, which hypothesizes that the function of dreaming is to "contextualize a dominant emotion of the dreamer," making "connections more broadly than waking in the nets of mind" (Hartmann, 1996). All these considerations are in agreement with Domhoff's assertion that "dreams express several key aspects of people's conceptual systems, especially self conceptions and conceptions of family and friends" (Domhoff, 2003).

Some limits of our study should be noted, mainly related to the small sample of patients, which is a consequence of difficulties in recruitment reported in earlier studies (Shover et al., 1995) in this research area.

The lapse of time of 3 months after mastectomy is supposed to be sufficient to allow for the disclosure of possible variations in dream content, given that the preceding literature (Anllo, 2000; Carver et al., 1998; Ghizzani et al., 1995; Henson, 2002; Shover et al., 1995) suggests that the appearance of a psychosexual crisis would not take place either before 2 or 3 months from the operation or after 12 months.

We did not consider breast prosthesis reconstruction, which could affect some analyzed variables. Some authors (Harcourt \& Rumsey, 2001) have underlined that a reconstructed breast has neither the function nor the physiological attributes of a natural breast because reparative surgery is not a perfect breast reconstruction. Moreover, Parker et al. (2007) demonstrated that psychosocial adjustment and quality of life are similar amongst patients with mastectomies with or without immediate reconstruction and patients undergoing breast conservative therapy.

Some concerns should be addressed regarding the home recall method versus a laboratory method. It could be noted that even though in-laboratory recall can increase the number of dreams (given the awakenings from each REM period), home recall allows subjects to fill in the questionnaire in a protected setting and make the written dream reports rich and articulated, as documented by result richness. Moreover, Domhoff's studies (Domhoff, 1996; Domhoff \& Schneider, 1998; Domhoff \& Schneider, 1999) provide evidence that "useful dream samples for studies using the Hall and Van de Castle (1966) coding system can be collected both in the laboratory or from normal recall at home" without significant differences in dream content (Domhoff \& Schneider, 1999, p. 139). However, home reports may be less artificial and are less expensive to collect than in-laboratory procedures (Hobson, 1992).

In conclusion, our study confirms the hypothesis that dreams indeed seem to reflect waking life concerns, and it reveals that a mastectomy produces body and self-concept modifications that are evident in a patient's dream content 3 months following surgery. The expression of fears and problems through dreams could help people who have difficulties to talk about what they really feel and how the experience of cancer is changing their conceptions about their bodies, themselves, the people around them, and their lives. Such information would significantly improve psycho-oncologists' ability to support their patients during their therapeutic course. 


\section{REFERENCES}

American Academy of Sleep Medicine. (2005). International classification of sleep disorders: Diagnostic and coding manual (2nd ed.). Westchester, IL: Author.

American Psychiatric Association. (2000). Diagnostic and statistical manual of mental disorders (4th ed., Text rev.). Washington, DC: Author.

Anllo, L. M. (2000). Sexual life after breast cancer. Journal of Sex and Marital Therapy, 26, 241-248.

Beck, A. T., Rush, A. J., Shaw, B. F., \& Emery, G. (1987) Cognitive Therapy of depression. Florence, Italy: Organizzazioni Speciali Press.

Brugger, P. (2008). The phantom limb in dreams. Consciousness and Cognition, 17, 1272-1278. doi: 10.1016/j.concog.2008.01.005

Carver, C. S., Pozo-Kaderman, C., Price, A. A., Noriega, V., Harris, S. D., Derhagopian, R. P., ... Moffatt, F. L. (1998). Concern about aspects of body image and adjustment to early stage breast cancer. Psychosomatic Medicine, 60, 168-174.

Domhoff, G. W. (1996). Finding meaning in dreams: A quantitative approach. New York, NY: Plenum Press.

Domhoff, G. W. (2001). A new neurocognitive theory of dreams. Dreaming, 11, 13-33. doi:10.1023/A: 1009464416649

Domhoff, G. W. (2003). The scientific study of dreams: Neural networks, cognitive development, and content analysis. Washington, DC: American Psychological Association Press. doi:10.1037/10463000

Domhoff, G. W. (2007). Dreaming as the embodiment of thoughts: A widower's dreams of his deceased wife. Paper presented at the annual meeting of the International Association for the Study of Dreams, Rohnert Park, CA. Retrieved on www.dreamresearch.net

Domhoff, G. W., Meyer-Gomes, K., \& Schredl, M. (2006). Dreams as the expression of conceptions and concerns: A comparison of German and American college students. Imagination, Cognition and Personality, 25, 269-282. doi:10.2190/FC3Q-2YMR-9A5F-N52M

Domhoff, G. W., \& Schneider, A. (1998). New rationales and methods for quantitative dream research outside the laboratory. Sleep, 21, 398-404.

Domhoff, G. W., \& Schneider, A. (1999). Much ado about very little: The small effect sizes when home and laboratory collected dreams are compared. Dreaming, 9, 139-151. doi:10.1023/A: 1021389615347

Fantini, M. L., Corona, A., Clerici, S., \& Ferini-Strambi L. (2005). Aggressive dream content without daytime aggressiveness in REM sleep behavior disorder. Neurology, 65, 1010-1015. doi:10.1212/ 01.wnl.0000179346.39655.e0

Ghizzani, A., Pirtoli, L., Bellezza, A., \& Velicogna, F. (1995). The evaluation of some factors influencing the sexual life of women affected by breast cancer. Journal of Sex and Marital Therapy, 21, 57-67.

Hall, C. S. and Van de Castle, R. L. (1966). The content analysis of dreams. New York, NY: AppletonCentury-Crofts.

Harcourt, D., \& Rumsey, N. (2001). Psychological aspects of breast reconstruction: A review of the literature. Journal of Advanced Nursing, 35, 477-487. doi:10.1046/j.1365-2648.2001.01865.x

Hartmann, E. (1996). Outline for a theory on the nature and functions of dreaming. Dreaming, 6, $147-170$.

Henson, H. K. (2002). Breast cancer and sexuality. Sexuality and Disability, 20, 261-275. doi:10.1023/ A:1021657413409

Hobson, J. A. (1992). The dreams machine. Florence, Italy: Giunti Press.

Mulder, T., Hochstenbach, J., Dijkstra, P. U., Geertzen, J. H. B. (2008). Born to adapt, but not in your dreams. Consciousness and Cognition, 17, 1266-1271. doi:10.1016/j.concog.2007.04.001

Newton, P. M. (1970). Recalled dream content and the maintenance of body image. Journal of Abnormal Psychology, 76, 134-139. doi:10.1037/h0029666

Parker, P. A., Youssef, A., Walker, S., Basen-Engquist, K., Cohen, L., Gritz, E. R., .. Robb, G. L. (2007). Short-term and long-term psychosocial adjustment and quality of life in women undergoing different surgical procedures for breast cancer. Annals of Surgical Oncology, 14, 3078-3089. doi:10.1245/s10434-007-9413-9

Revonsuo, A. (2000). The Reinterpretation of Dreams: An evolutionary hypothesis of the function of dreaming. Behavioral and Brain Sciences, 23, 877-901. doi:10.1017/S0140525X00004015

Rezzonico, G., \& Liccione D. (2004). Dreams and Psychotherapy: the use of dream material in cognitive psychotherapy. Torino, Italy: Bollati Boringhieri Press.

Rumsey, N., \& Harcourt, D. (2004). Body image and disfigurement: Issues and interventions. Body Image, 1, 83-97. doi:10.1016/S1740-1445(03)00005-6

Schneider, A. (1995). DreamSAT. Retrieved December 5, 2011 from the World Wide Web: http:// dreamresearch.net/DreamSAT/ 
Sheppard, L. A., \& Ely, S. (2008). Breast cancer and sexuality. The Breast Journal, 14, 51-56. doi: $10.1111 / \mathrm{j} .1524-4741.2007 .00550 . \mathrm{x}$

Schover, L. R., Yetman, R. J., Tuason, L. J., Meisler, E., Esselstyn, C. B., Hermann, R. E., . . Dowden, R. V. (1995). Partial mastectomy and breast reconstruction. Cancer, 1995, 75, 54-64.

Spielberger, C. D. (1989). STAI State-Trait Anxiety Inventory (form Y). Florence, Italy: Organizzazioni Speciali Press.

Vetrugno, R., Arnulf, I., \& Montagna, P. (2008). Disappearance of "phantom limb" and amputated arm usage during dreaming in REM sleep behavior disorder. Journal of Neurology, Neurosurgery, and Psychiatry, 79, 481-483. doi:10.1136/jnnp.2007.129783

Walsh, S. R., Manuel, J. C., \& Avis, N. E. (2005). The impact of breast cancer on younger women's relationships with their partner and children. Families, Systems, and Health, 23, 80-93. doi:10.1037/ 1091-7527.23.1.80 